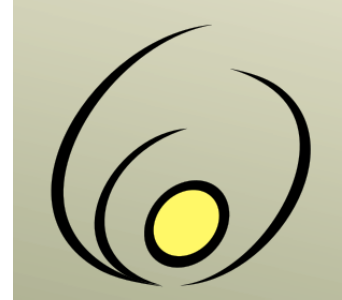

Fórum de Pró-Reitores de Extensão das Instituições Públicas de

Educação Superior Brasileiras

\title{
Actividad física y adultos mayores: Contribución desde la extensión universitaria
}

\author{
Adriana San Martín ${ }^{1}$, Fernanda Américo ${ }^{2}$, Artur Bonezi ${ }^{3}$, Renata Luísa Bona ${ }^{4}$
}

Resumen: El objetivo del este estudio fue evaluar la promoción la práctica de actividad física realizada durante un semestre junto a un grupo de adultos mayores, así como los efectos de la interacción entre docentes estudiantes y adultos mayores. Luego después de 25 semanas de actividades prácticas, fue propuesto un cuestionario abierto pensamientos de adultos mayores, el equipo de docentes y de estudiantes involucrados. La propuesta alcanzo sus objetivos, prevención y promoción de salud a los adultos mayores, el desarrollo de una actividad práctica y experiencia profesional a los estudiantes, así como el intercambio entre los universitarios/as y los adultos mayores creando un espacio de formación integral e interdisciplinario.

Palabras-clave: Vejez; Movimiento; Comunidad

\section{Physical activity and older adults: Contribution from university extension}

Abstract: The objective of this study was to evaluate the promotion of the practice of physical activity carried out during a semester with a group of older adults and the effects of the interaction between student teachers and older adults. After 25 weeks of practical exercises, an evaluation with an open questionnaire was proposed. The results obtained were about the sensations, emotions, and thoughts of the elderly, the teaching, and the student teams involved. The proposal reached its objectives: prevention and promotion of health to the elderly, development of practical activity and professional experience for students, an exchange between the actors of the university public and the elderly creating a comprehensive and interdisciplinary formation space.

Keywords: Elderly; Movement; Community

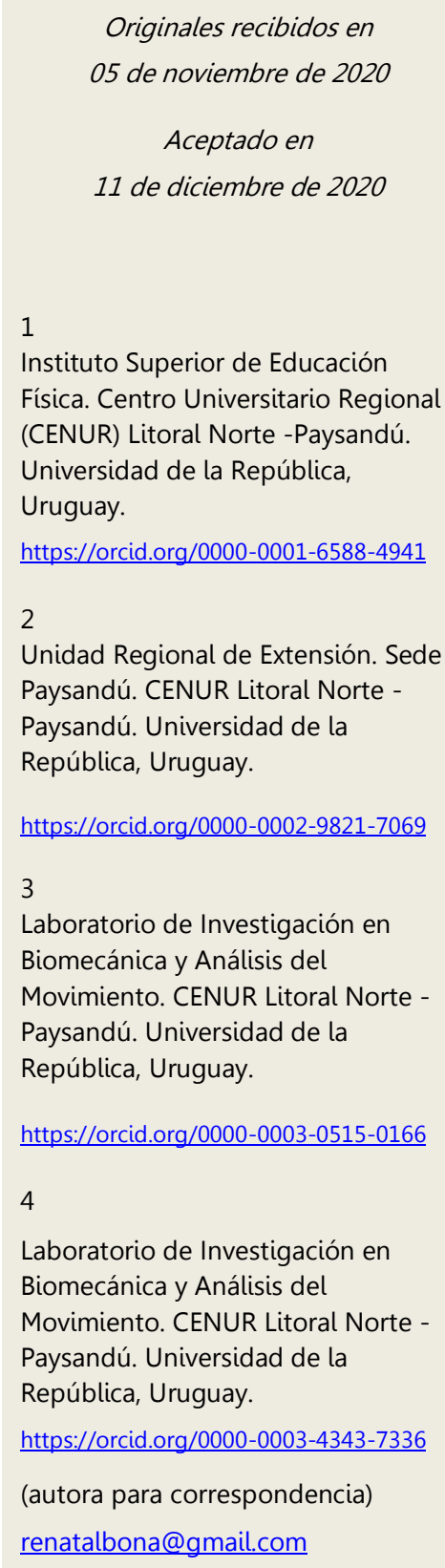




\section{Introducción}

El envejecimiento y sus pronosticadas consecuencias sociales son temas de creciente preocupación. Por ello, se ha sugerido desde las diversas disciplinas sociales impulsar nuevas líneas de investigación en torno a las diferentes actividades practicadas entre las personas mayores, como actividad física (AF) y deportivas (Del Castillo et al., 2006).

La práctica de AF posee relación con las experiencias pasadas y estilos de vida, condicionan las actuaciones futuras en AF (Levinson, 1978; Vuolle, 1985; Lehr, 1999). Según la Organización Mundial de la Salud (2018), la $\mathrm{AF}$ es reconocida como un elemento fundamental para el mantenimiento y construcción de un individuo saludable.

En este sentido, las personas adultas que participan en AF muestran razones más asociadas al factor social (Moreno-Murcia et al., 2008). Motivos como estar con los amigos, conocer a gente nueva, o compartir el tiempo, estarían dentro del dominio de afiliación social como una de las metas más importantes de la práctica físico-deportiva, promoviendo una buena calidad de vida y así un equilibrio bio-psíco-social.

Uruguay es uno de los países más envejecidos del contexto latinoamericano con un alto índice de adultos mayores ( $A D$ ). La mayoría de estos viven de forma sedentaria o carecen de centros con profesionales que lleven adelante prácticas de gimnasias adaptadas, teniendo en cuenta además todo lo que conlleva el envejecimiento desde un aspecto físico y social. Uruguay tiene un programa dentro de Ministerio de Desarrollo Social, el Instituto Nacional de las Personas Mayores-Inmayores (ley n 18.617, 2012, "Plan Nacional de Envejecimiento y Vejez"). Tiene como fin la promoción integral de $A D$ y sus funciones son el diseño, la coordinación y evaluación de las políticas sociales dirigidas a esta población. Asimismo, se creó el Plan Nacional de Envejecimiento y Vejez, el cual es el primer plan gerontológico de Uruguay. El Estado asume niveles de compromiso y un rol para la sociedad civil, en acciones políticas y participación en línea de abordaje de trabajo.

Con esto, desde la Universidad de la República de Uruguay (UdelaR), sentimos la importancia de la extensión universitaria y su impacto en este grupo social. Dentro de las actividades de extensión universitaria es posible interactuar con diferentes grupos, así como con los AD. Algunos de estos suelen aislarse, por miedo a la inseguridad que conlleva la vida moderna, desarrollando problemas físicos, posturales, malos hábitos, el sedentarismo, entre otros. Esto lleva al surgimiento de complicaciones a nivel psíquico y cognitivo, así como problemas como la depresión y la ansiedad. En consecuencia de todo este proceso que lleva el aislamiento, es difícil para el $A D$ volver a insertarse en la sociedad, y los familiares son la base para que todo lo nombrado sea evitado (López, \& Clemente, 2019). Para esto existen instituciones como CEUPA/UNI3 (Centro de Estudios Universitarios Permanentes y Abiertos/ Universidad de la Tercera Edad), la cual ofrece talleres abiertos para todas las edades, inclusive $A D$, motivándolos a seguir vivenciando diferentes experiencias. Este tipo de institución es parte formal de la UdelaR, lo que torna posible desarrollar actividades de extensión universitaria, generando un espacio de vínculo y actividad entre sociedad, estudiantes y docentes.

La propuesta planteó como objetivo general, conocer los aportes y beneficios de la AF realizada en grupo una vez a la semana, en relación a la promoción y prevención de la salud en AD. Los objetivos específicos fueron: i) Realizar ejercicios básicos para el equilibrio y postura en AD, ii) Generar vínculo con la organización CEUPA para avanzar hacia la interacción entre AD y Universidad.

Para alcanzar los objetivos propuestos, el eje central fue la movilidad física y prevención de lesiones en $A D$, involucrando el Laboratorio de Investigación y Análisis del Movimiento (LIBiAM), la pasantía para estudiantes de Instituto Superior de Educación Física (ISEF) y el grupo de AD de la organización CEUPA, con el apoyo y 
acompañamiento de la Unidad Regional de Extensión de la Sede Paysandú CENUR Litoral Norte de la Universidad de la República.

¿Qué aspectos implicaron el desarrollo de esta propuesta pensada como proceso a construir? La presentación de la propuesta a todos los involucrados y conocernos para comenzar; realizar un acercamiento a la comprensión de la Extensión Universitaria; la organización del trabajo colectivo; la integración de aspectos curriculares de estudiantes y docentes de la disciplina educación física. La planificación y puesta en marcha de actividades y espacios de encuentro para diagnóstico inicial, formación, aspectos teóricos, charlas informativas, ejercicios prácticos y evaluación.

\section{Métodos}

El estudio posee un diseño cualitativo. Después del período de actividades prácticas desarrolladas por los estudiantes y docentes junto a los $A D$, fue propuesto un cuestionario abierto a modo de evaluación (Material Suplementario). El presente trabajo es basado en las respuestas obtenidas en el cuestionario tanto para los $A D$, estudiantes y docentes, teniendo como fin conocer las reflexiones, sensaciones, emociones y pensamientos de forma particular de cada participante de la actividad de extensión desarrollada. Se abordó una problemática de interés social en forma interdisciplinaria involucrando docentes del LIBiAM, y estudiantes de ISEF.

Las actividades prácticas fueron organizadas durante seis meses, una vez en la semana, durante 60 min, un total de 25 encuentros durante el año, de la siguiente forma:

\section{Descripción de las actividades físicas desarrolladas durante el año}

La finalidad principal de la actividad de extensión era la movilidad física, prevención de lesiones y el acercamiento del estudiante con la práctica de AF con AD. Las clases estaban elaboradas según un cronograma semestral creado en conjunto por estudiantes y docentes, adaptado a las características, capacidades y patologías que presenta cada AD. Los encuentros se realizaron en salones y en la pista de atletismo del complejo deportivo y educativo de Paysandú, que conjuga recursos de la Intendencia Departamental, la UdelaR, el Consejo de Educación Técnico Profesional y la Secretaría Nacional del Deporte, en la Sede Paysandú del CENUR Litoral Norte, que dispone de una estructura física de aulas, laboratorios y oficinas. Allí se encuentra recientemente inaugurado el LIBiAM del CENUR Litoral Norte de la UdelaR.

Las actividades eran compuestas de circuitos funcionales por estaciones, adaptadas a todas las limitaciones que presentaban los $A D$ asistentes. Los estudiantes y docentes trataban de incentivar las ejecuciones de los ejercicios seleccionados con calidad de movimiento, buscando mejorar la postura y previniendo futuras lesiones.

Para las clases planificadas se tuvo en cuenta los beneficios, recomendaciones y progresiones de ejercicios específicos para AD según él American College Sports Medicine (ACSM) (Chodzko-Zajko et al., 2009) y American Heart Association (AHA) (Nelson et al., 2007). La programación de ejercicios físicos para las personas mayores, según la literatura citada, consiste en un entrenamiento supervisado, en este caso por los estudiantes y docentes, asesoramiento y prescripción del ejercicio, seguido de un programa controlado por un profesional y programación de ejercicio con base en la propia comunidad, con dirección y control. Las jornadas de encuentro eran controladas por los estudiantes, haciendo un asesoramiento e hincapié en la postura, que tiende a retroceder por malos hábitos cotidianos, avance de la edad, y ejecución de los ejercicios que realizaban los adultos (Feijó et al., 2018). Antes y después de las clases con los AD los docentes tenían espacio para discutir dudas y lo que fue realizado en el día, y construir las rutinas que serían suministradas en la clase siguiente. 


\section{Descripción de las evaluaciones finales}

Los $A D$, docentes y estudiantes contestaron a través de cuestionarios abiertos. Durante las respuestas todos podrían preguntar respecto a dudas en cuanto a las preguntas. El cuestionario tenía una división en siete aspectos: i) Cumplimiento de objetivos y construcción de la propuesta (¿Qué se quería lograr? ¿Hubo participación de toda/os en la construcción y planificación de la propuesta? ¿Se alcanzaron acuerdos de trabajo?); ii) Estrategia de intervención (¿Qué pasos se dieron para alcanzar los objetivos? ¿Qué dificultades se presentaron? ¿Cómo evalúan el vínculo construido con las personas integrantes de la organización CEUPA que participaron de la experiencia?); iii) Organización del equipo (¿Qué dificultades/fortalezas existieron en la organización del trabajo? ¿Cuáles fueron las fortalezas/debilidades de la formación disciplinar en el proceso de desarrollo de la propuesta? ¿Cómo se sintieron en los diferentes momentos del proceso?); iv) Sobre la orientación docente (¿Cómo evalúan el rol de los/as docentes responsables de la propuesta?); v) Resultados (¿Cuáles fueron los resultados alcanzados? ¿Cuáles consideran que fueron los principales aprendizajes del equipo?); vi) Proyección (¿Se abrieron nuevas perspectivas de profundización de la línea de trabajo? ¿Se vinculan a actividades de enseñanza, extensión o investigación?); para finalizar vii) Comentarios y sugerencias. Las respuestas fueron señaladas en cuanto a sus características semejantes (dentro de cada grupo: estudiantes, docentes, $A D$ ), y a partir de este análisis fue propuesto el desarrollo de este trabajo en formato de resultados y discusión.

El estudio siguió las normas éticas de la Declaración de Helsinki. La presente intervención pose aprobación del comité de ética de la UdelaR, número de expediente: 311110-000182-17. Todos los sujetos participantes firmaron el consentimiento informado.

\section{Resultados}

En las últimas instancias del proceso, fueron realizadas una actividad de cierre y de evaluación, recogiendo percepciones desde la organización CEUPA, el equipo docente y el equipo estudiantil, incorporando la concepción metodológica de la sistematización de experiencias, con la coordinación de la Unidad de Extensión ${ }^{1}$.

Con la experiencia de las clases y con las respuestas de los cuestionarios, fue posible obtener los siguientes resultados, desarrollados a continuación en los siguientes subtítulos: estudiantes, docentes y adultos mayores (AD).

\section{Estudiantes}

A través del espacio brindado por CEUPA para que los AD puedan realizar AF en forma de taller, se pudo trabajar las diferentes capacidades físicas, como coordinación (gruesa y fina), equilibrio corporal, agilidad, fuerza, resistencia, potencia muscular y el lúdico, para un mejoramiento de la calidad de vida de los participantes. También se generó un espacio para socializar-se, más allá de la AF, proporcionando el bienestar de todos.

Semanalmente, se trabajó en conjunto en la planificación y la ejecución de las clases, así como también las mismas estaban divididas por capacidades físicas. Tanto en la planificación como para la clase, se generó buena comunicación entre todos los involucrados, y en el desarrollo de las clases cada estudiante pudo aportar sus conocimientos. 
Para alcanzar el objetivo se investigó acerca de las características y limitaciones que presentan los $A D$, a través de toda la información recopilada en todas las jornadas planificadas. El vínculo constituido entre estudiantes, tutores y $A D$ fue cálido, en un relacionamiento abierto de expresión grupal, sin roles estereotipados.

Con relación a los aspectos considerados difíciles fueron citados, la inexperiencia para trabajar con el público mayor y la falta de conocimiento e información académica acerca de los cambios que sufre el "cuerpo adulto", en relación a la anatomía, fisiología, cambios biológicos que sufre una persona con el avanzo de la edad, así como también patologías comunes en esta franja etaria. Los estudiantes realizaron búsquedas científicas y presentaciones a los docentes sobre temas del envejecimiento como, alteraciones fisiológicas, musculoesqueléticas, articulares, biomecánicas, sistema nervioso. Otro aspecto considerado difícil fue el espacio e infraestructura, lo cual era compartido con otras disciplinas, el espacio no estaba adaptado a la población. Por algunos momentos del proceso los nervios, la inseguridad estaban presentes, así como también la incertidumbre hacia la reacción de los participantes.

Lo que refiere a las fortalezas en la organización, se señaló el interés de parte de todos, una buena comunicación tanto de parte de los tutores, estudiantes, y AD. Otra fortaleza fue el respaldo del docente (el tutor) desde sus experiencias previas y conocimientos académicos, también destacando las devoluciones y críticas constructivas luego de culminar cada clase. Sin embargo, las dificultades/ fortalezas de la formación disciplinar estaban relacionadas con la falta de información brindada por la carrera académica en relación a la anatomía, fisiología, los cambios biológicos que sufre una persona con el avanzo de la edad, así como también las patologías que son comunes para esta franja etaria. Con respecto a la formación disciplinar, se destaca la experiencia previa de "como posicionarse frente al grupo o frente a una clase", la buena voluntad de parte del grupo, la creatividad a la hora de presentar propuestas. También fueran consideradas importantes las búsquedas y presentaciones de los estudiantes solicitada por los tutores con relación a temas alteraciones fisiológicas, musculoesqueléticas, articulares, biomecánicas y también relacionadas con el sistema nervioso.

Por otra parte, los resultados fueron alcanzados, se generó un vínculo dentro del espacio intergeneracional entre todos los involucrados de diferentes franjas etarias, nuevos conocimientos obtenidos desde los ejercicios adaptados, se fortaleció la didáctica, y se abrieron nuevas perspectivas de profundización.

Para dar secuencia a las actividades, las sugerencias fueron implementar una carga horaria mayor, así como la articulación con otros departamentos del ISEF y otras disciplinas. También poder profundizar el enfoque de la formación en extensión e integralidad universitaria.

\section{Docentes}

En cuanto a la organización y coordinación de las propuestas, las clases prácticas fueran consideradas amenas, con una buena interacción con los estudiantes y AD.

Fue difícil la organización de los materiales, debido al volumen de los mismos, así como por los espacios compartidos. Además, en un principio los estudiantes no tenían conocimiento de los cambios relacionados al envejecimiento. Asimismo, el cambio de lugar de encuentro, de la sede central para el polideportivo, dificultó la participación de algunos AD. El traslado que debían realizar los AD complicó al principio la participación de algunos.

El relacionamiento, participación e interacción de los involucrados fue buena entre todos, los factores que facilitaron fueron el entusiasmo y la buena disposición de los AD y el interés de los estudiantes.

Fue posible cumplir todos los objetivos planteados en un principio, por parte de los docentes a cargo. En cuanto a la metodología empleada, para cada clase práctica rotaba el estudiante responsable, se utilizaba mayoritariamente los materiales adquiridos para los ejercicios físicos con los $A D$. 
Se resalta el fortalecimiento de la actividad "pasantía" con ISEF, también, la integración "taller" con CEUPA. Así mismo, hubo diferentes niveles de participación desde la teoría (presentaciones de temas respecto al envejecimiento por parte de los estudiantes) hasta la práctica. Sentimientos asociados a la práctica: gratificante, muy importante y bueno interactuar con $A D$, a la vez, orientar otras personas, estudiantes, a desarrollar esta actividad, contribuyendo a su formación profesional.

\section{Adultos mayores}

En cuanto a las vivencias en las clases, los $A D$ destacaron, "los ejercicios físicos eran realizados en conjunto y con alternancia de momentos de relajación". Además, fue explicado cómo realizar los movimientos cuidando siempre la columna, la manera correcta de caminar para no tropezar. La importancia de la respiración, de los estiramientos, también fueron planteadas. Fueron aportados diferentes consejos de los profesores y estudiantes, en cuanto a la coordinación, prevención, los cuales fueron muy buenos, bien adaptados a la franja etaria de los participantes. Las actividades generaron un espacio de confianza de inserción a todas las actividades.

En cuanto a la forma de organización del trabajo las respuestas fueron positivas: "las actividades prácticas diversas y alegres, excelente; organizado, supervisado por docentes pendientes en todo momento, haciendo las correcciones necesarias; en equipos con un estudiante a cargo de la clase; buena metodología, planificación y variación de las propuestas; con atención individualizada, corrección personalizada". "Se logró generar un ambiente armonioso y un vínculo estable." (relató un adulto mayor). Consignas claras, manteniendo la amabilidad y respeto hacia las personas mayores. Se visualizaban diferentes manifestaciones de participación en las actividades. Se pudo cumplir todas las expectativas de integración y el principal resultado positivo remarcado por los $A D$ es en cuanto a la postura física.

El proyecto de extensión aportó desde la promoción y prevención de la salud, se pudo afirmar conceptos ya existentes y dar a conocer otros, esto se tomó como un factor muy positivo para todos los participantes. Reforzamos la concientización respecto a la movilidad y la importancia de realizar AF regularmente. Los cuidados planteados en las clases hacen hincapié en su interés en continuar con la propuesta a través de los espacios de taller de CEUPA. Les resulta muy interesante e importante la prolongación de este espacio y que el mismo cuente con instancias tanto prácticas como teóricas, explicando ejercicios (cómo se hacen y para qué sirven).

Los $A D$, cuando refieren a cómo se sintieron en las experiencias desarrolladas, describen sus "ganas de venir siempre", muy motivadora e integradora, se sienten a gusto principalmente cuando se realizan las actividades al aire libre. Describen sentimientos como de apoyo, protección, a gusto, agradecimiento "a la vida". Consultados respecto a las dificultades en el proceso, no manifiestan ninguna dificultad, por el contrario, refuerzan la atención recibida de parte de los tutores y estudiantes, uno manifiesta la cuestión del horario y otro, la patología que lo limita en ciertos ejercicios, pero refuerza que es muy ayudado por todos.

En cuanto a los desafíos de la propuesta, la "exigencia" para lograr una mejor calidad de vida es apuntada y también, algunos ejercicios que, sin embargo, fue posible adaptar y realizar. Muchos AD destacan el logro de priorizar la salud y mejorar costumbres como la mala postura (hipercifosis torácica o hiperlordosis lumbar y/o cervical), tomar conciencia de "mi cuerpo" y sus posibilidades y limitaciones, "mejorar el carácter para actuar en la sociedad". Las manifestaciones en cuanto al aprendizaje considerado más importante, refieren al estar atento al propio cuerpo y corregir mediante lo aprendido, destacan "el hecho de haber caminado por la pista de atletismo, fue lo mejor", "sin AF no hay bienestar", el respeto hacia el prójimo, el cuidado y autocuidado.

Acerca de cómo quisieran proyectar y dar continuidad al taller, los AD sugieren proseguir el siguiente año, con más días y horarios, más meses de dictado de taller, profundización en algunos aspectos teóricos, actividades 
al aire libre. Cerrando, destacan la importancia de cada participante y palabras de agradecimiento fueran muy acentuadas.

\section{Discusión}

El presente estudio alcanzó su objetivo inicial, de observar y estudiar la práctica de AF realizada durante un semestre junto al grupo de $A D$, generando interacción y espacio para el aprendizaje entre estudiantes de ISEF, docentes y AD (CEUPA).

Las características solicitadas inicialmente, fueron desarrolladas en base a la organización y planificación para las clases de AF. Las planificaciones por parte de los estudiantes, estaban respaldadas por el conocimiento y experiencia de los tutores. Así mismo, brindaban un soporte como la revisión de las clases, conceptos básicos de AF con AD, discusión en cuanto a dudas, entre otros (De Mantrana, 2005; Chacón Corzo, 2007). De forma general las respuestas brindadas por los diferentes grupos en las evaluaciones posibilitaron conocer los aspectos positivos, debilidades y formas de mejorar la actividad para su seguimiento.

Los estudiantes relataron que, desde sus reflexiones, sus objetivos en cuanto a la propuesta, fueron alcanzados. A través de la interacción entre docentes y $A D$ fue logrado un aprendizaje teórico y práctico. Las actividades prácticas organizadas por los estudiantes de ISEF generó una búsqueda de conocimiento, en primera instancia de forma teórica y luego de forma práctica. El vínculo entre docentes y estudiantes resultó de gran aprovechamiento con discusiones de temas específicos del envejecimiento, cuidados con la postura, lesiones y enfermedades comunes a la tercera edad. Salir de los textos, discutir esta información y luego poner en práctica fue extremamente positivo (De Mantrana, 2005). La estrategia de estar actuando con supervisión de forma confortable y segura, practicar lo que será en breve su profesión, discutir los temas en equipo permitió vivenciar una forma de trabajo en equipo positiva. Expresan la voluntad de seguir en la actividad, además de sentir la necesidad de interactuar y conocer más en torno a la extensión universitaria, resultado del trabajo positivo planteado (De Mantrana, 2005). Generar formas de involucrar a los estudiantes, a través de la coordinación del curso, así como con otros docentes del ISEF, es uno de los objetivos del equipo que propuso esta actividad.

Las prácticas de extensión comprenden varias lógicas de intervención y de comprometerse con el momento histórico que atraviesa nuestra sociedad (Cataldo, 2012). Los tutores han sido los conductores del acercamiento del estudiante universitario de ISEF con la comunidad de AD. Las jornadas tuvieron una buena interacción, por ese motivo se pudo cumplir todos los objetivos planteados. La organización establecida fue de forma grupal, pero la responsabilidad fue más individual para cada jornada práctica, con una orientación previa. En las jornadas teóricas, acerca los temas específicos (Chodzko-Zajko et al. 2009; Nelson et al. 2007; Feijó et al., 2018), se armaban grupos de discusión para comprender mejor los cambios que trae el envejecimiento (Filpe et al., 2008). La práctica desarrollada presentó reflexión personal y de grupo. Los avances visualizados en los estudiantes y $A D$, así como el vínculo generado, son aspectos positivos que generan fuerza para seguir con la actividad (Schön, 1988). La práctica profesional y la docencia son actividades que retroalimentan. Entendemos que la práctica, discusiones, experiencias con su profesión, generarán profesionales actualizados e inserción rápida en su área (Filpe et al., 2008; Scalabrin \& Molinari, 2013). La actividad generó gratitud, culminando el proyecto de extensión, destacando su importancia y la de interactuar con AD y orientar a estudiantes. Las vivencias con la tercera edad, escuchar sus experiencias, sus dificultades, hacen pensar respecto de este grupo en nuestra sociedad. Los estudiantes con sus inseguridades, dificultades, energía y disposición suman fuerza a la actividad. La vinculación entre AD y estudiantes genera aprendizajes para diferentes áreas de la vida. 
Santos y cols. (2014) reportaron en su estudio, cual involucró la actividad de extensión y práctica de AF en AD, que el ocio pose asociación de forma positiva con características relacionadas al psicológico (autoestima, la espiritualidad, la imagen corporal y el pensar y aprender), concluyendo que la variedad de actividades de ocio puede resultar en una buena aptitud mental, favoreciendo una percepción positiva de la vida en términos psicológicos. Otro estudio que también presentó relación entre AF y la actividad de extensión universitaria, mostró la relación cuanto a práctica de AF y la percepción de calidad de vida en AD (Kretzer et al., 2011). Combinando AF y extensión, pero buscado control de los niveles del diabetes, Ribeiro y cols. (2017) presentaron resultados positivos con la intervención en AD. Enfatizan la necesidad de mayor cantidad de programas y mayor difusión.

Las prácticas de los ejercicios físicos adaptados para los AD aportaron al cumplimiento de los objetivos propuestos, tomando estos de una forma positiva hacia el abandono del sedentarismo que comúnmente está presente en esta población especial. A través de diferentes estudios conocemos los prejuicios de la inactividad en $A D$, que van desde el emocional (Yasunaga et al., 2018) hasta diferentes aspectos fisiológicos (Wullems et al. 2016). Las actividades facilitaron la comunicación e integración del grupo, y las mismas contenían variación para no ser algo rutinario/repetitivo y cerrado. Las actividades en grupo generan diferentes beneficios a los AD, más allá de físicos, también cognitivos y emocionales (Assari, 2017; Leires-Rodríguez et al., 2018). Los resultados alcanzados estaban relacionados con lo físico, ya que los adultos afirman una mejora en su postura y aptitud para realizar tareas. También, relacionadas con lo psíquico y social, condicionalmente una de las finalidades era mejorar la integración y comunicación y consecuentemente el proceso de socialización, generando un espacio de confianza (Mabli et al., 2017). Se manifiesta el interés de seguir con espacios como el formado, quizás con un público mayor y más carga horaria, para que los encuentros sean más frecuentes durante la semana y al aire libre.

\section{Conclusión}

La propuesta planteada pretendió aportar a la promoción y prevención de la salud en AD desde el intercambio entre universitarios y la organización CEUPA. Los resultados fueron positivos y corroboran con estudios de otros países y la necesidad de implementación de más actividades con perfil semejante. El vínculo establecido entre los $A D$, estudiantes y docentes fue positivo, de acuerdo con las respuestas obtenidas. Además de generar confianza entre los involucrados, los beneficios de práctica orientada a los estudiantes resulto en experiencia profesional, y en los $A D$ una evolución relatada desde lo psíquico y social, bien como del postural y equilibrio.

El giro cognoscitivo qué propone la integralidad universitaria se concibe desde una perspectiva que significa pensar en circunstancia, habilitando el ejercicio de la reflexividad.

\section{Contribución de cada autor}

A.S.M. participó de proyecto, redacción del artículo, aplicación de los métodos; F.A participó del protocolo, redacción del artículo; F.A, R.L.B y A.B planearon el proyecto, R.L y A.B aplicación de los protocolos, análisis de los datos e interpretación, redacción del artículo y revisión intelectual crítica.

\section{Notas}

1. Se trata de una herramienta de registro, escritura, interpretación, análisis, que proviene de la metodología conocida como Sistematización de Experiencias, surgida en América Latina a partir de los años 1960, como producto del esfuerzo por construir marcos propios de interpretación teórica desde las condiciones particulares de nuestra realidad. 
Nos propone conocer nuestras percepciones y significaciones, teniendo en cuenta el hacer, el pensar y el sentir en relación a la experiencia desarrollada, como ejercicio de producción de conocimiento crítico y transformador desde la práctica, permitiéndonos valorarla, mejorar nuestras prácticas y darle proyección a la propuesta con las miradas y los aportes de toda/os quienes fuimos parte (Jara, 2013).

\section{Referencias}

Assari, S. (2017). Association between self-esteem and depressive symptoms is stronger among black than white older adults. Journal of Racial and Ethnic Health Disparities, 4(4), 687-695.

Cataldo, A. E. (2012). Extensión universitaria y educación física. Extensión en Red, (3).

Chacón Corzo, M. A. (2007). La enseñanza reflexiva en la formación de los estudiantes de pasantías de la carrera de Educación Basica Integral. Tarragona: Universitat Rovira i Virgili.

Chodzko-Zajko, W. J., Proctor, D. N., Singh, M. A. F., Minson, C. T., Nigg, C. R., Salem, G. -J., \& Skinner, J. S. (2009). American College of Sports Medicine (ACSM) Position Stand: Exercise and physical activity for older adults. Medicine \& Science in Sports \& Exercise, 41(7), 1510-1530.

Del Castillo, J. M., Jiménez-Beatty, J. E., Graupera, J. L., \& Rodríguez, M. L. (2006). Condiciones de vida, socialización y actividad física en la vejez. Revista Internacional de Sociología, 64(44), 39-62.

De Mantrana, M. S. (2005). El aprendizaje en contextos laborales reales: El caso de las pasantías de los estudiantes universitarios. Educere, 9(30), 345-357.

Feijó, F., Bonezi, A., Stefen, C., Polero, P., \& Bona, R. L. (2018). Evaluación de adultos mayores con tests funcionales y de marcha. Educación Física y Ciencia, 20(3), 1-16.

Filpe, M. D. L. M., Guitelman, S., \& Abate, S. M. (2008). El aula: "Una conversación animada de límites imprecisos". Arte e Investigación, 12: 50-56.

Leirós-Rodríguez, R., Soto-Rodríguez, A., Pérez-Ribao, I., \& García-Soidán, J. L. (2018). Comparisons of the health benefits of strength training, aqua-fitness, and aerobic exercise for the elderly. Rehabilitation Research and Practice, 2018: 5230971.

Levinson, D. (1978). The seasons of man's life. New York: Alfred A. Knopp.

Lehr, U. (1999). El envejecimiento activo. El papel del individuo y de la sociedad. Revista Española de Geriatría y Gerontología, 34(6), 314-318.

López, E. R., \& Clemente, C. C. (2019). Soledad y aislamiento, barreras y condicionamientos en el ámbito de las personas mayores en España. Revista Internacional de Políticas de Bienestar y Trabajo Social, 12: 127-154.

Kretzer, F. L., Guimarães, A. C. D. A., Dário, A. B., Kaneoya, A. M., Tomasia, D. L., Feijó, I., Simas J. P. N., \& Parcias, S. R. (2011). Qualidade de vida e nível de atividade física de indivíduos na meia idade participantes de projetos de extensão universitária. Revista Baiana de Saúde Pública, 34(1), 146-158.

Jara, O. (2013). La sistematización de experiencias. Práctica y teoría para otros mundos posibles. Montevideo: EPPAL, Biblioteca de Educación Popular.

Mabli, J., Gearan, E., Cohen, R., Niland, K., Redel, N., Panzarella, E., \& Carlson, B. (2017). Evaluation of the effect of the Older Americans Act Title III-C Nutrition Services Program on participants' food security, socialization, and diet quality. Washington, DC: US Department of Health and Human Services, Administration for Community Living.

Moreno-Murcia, J. A., González-Cutre, D., \& Cervello-Gimeno, E. (2008). Motivación y salud en la práctica físicodeportiva: Diferencias según el consumo de alcohol y tabaco. International Journal of Clinical and Health Psychology, 8(2), 483-494. 
Nelson, M. E., Rejeski, W. J., Blair, S. N., Duncan, P. W., Judge, J. O., King, A. C., \& Castaneda-Sceppa, C. (2007). Physical activity and public health in older adults: Recommendation from the American Heart Association. Circulation, 116(9), 1094-1105.

Organización Mundial de la Salud (2018). Envejecimiento y salud. [S. I.]: OMS. Recuperado de https://www.who.int/es/news-room/fact-sheets/detail/envejecimiento-y-salud

Ribeiro, J. N. S., Lima, A. M. B., França, J. A. L., Silva, V. N. S., Cavalcanti, C. B. S., \& Vancea, D. M. M. (2017). Doce Vidaprograma de exercício físico supervisionado para diabéticos. Revista Andaluza de Medicina del Deporte (ahead of print version) Recuperado de: https://reader.elsevier.com/reader/sd/pii/S1888754616301344?token=1D923CBF 2A489BF28D9B0E07C857B37CA7481DCD62AE4CB877A559383969E85FD25983D4EF787899885279A22F5A7DC1

Santos, P., Marinho, A., Mazo, G., \& Hallal, P. (2014). Atividades no lazer e qualidade de vida de idosos de um programa de extensão universitária em Florianópolis (SC). Revista Brasileira de Atividade Física \& Saúde, 19(4), 494-503.

Scalabrin, I. C., \& Molinari, A. M. C. (2013). A importância da prática do estágio supervisionado nas licenciaturas. Revista Unar, 7(1), 1-12.

Schön, D. A. (1988). El profesional reflexivo: Cómo piensan los profesionales cuando actúan. Barcelona: Editorial Paidós. Vallejo, N. G. (2007). Actividad física y envejecimiento. Sevilla: Wanceulen SL.

Vuolle, P. (1985). Social motives as determinants of physical recreation at various life stages. En AIESEP Congress, An international dialogue on research on physical education and sport, Nueva York, Adelphi University.

Wullems, J. A., Verschueren, S. M., Degens, H., Morse, C. I., \& Onambélé, G. L. (2016). A review of the assessment and prevalence of sedentarism in older adults, its physiology/health impact and non-exercise mobility counter-measures. Biogerontology, 17(3), 547-565.

Yasunaga, A., Shibata, A., Ishii, K., Koohsari, M. J., \& Oka, K. (2018). Cross-sectional associations of sedentary behaviour and physical activity on depression in Japanese older adults: An isotemporal substitution approach. BMJ Open, 8(9), e022282.

Cómo citar este artículo:

San Martín, A., Américo, F., Bonezi, A., \& Bona, R. L. (2021). Actividad física y adultos mayores: Contribución desde la extensión universitaria. Revista Brasileira de Extensão Universitária, 12(1), 33-42. https://periodicos.uffs.edu.br/index.php/RBEU/article/ view/11755/pdf 